\title{
Is increased water consumption among older adults associated with improvements in glucose homeostasis?
}

\author{
Adrienne G. Clark ${ }^{1}$, Elizabeth A. Dennis Parker², Jyoti S. Savla ${ }^{3}$, Kevin P. Davy ${ }^{1}$, \\ Brenda M. Davy ${ }^{*}$ \\ ${ }^{1}$ Department of Human Nutrition, Foods and Exercise, Virginia Tech, Blacksburg, USA; *Corresponding Author: bdavy@vt.edu \\ ${ }^{2}$ Lombardi Comprehensive Cancer Center, Georgetown University Medical Center, Washington, D.C., USA \\ ${ }^{3}$ Department of Human Development, Virginia Tech, Blacksburg, USA
}

Received 25 May 2013; revised 26 June 2013; accepted 10 July 2013

Copyright (C) 2013 Adrienne G. Clark et al. This is an open access article distributed under the Creative Commons Attribution License, which permits unrestricted use, distribution, and reproduction in any medium, provided the original work is properly cited.

\section{ABSTRACT}

Obesity and impaired glucose homeostasis in older adults place these individuals at risk for diabetes. Dehydration, glucose homeostasis, and insulin resistance are related; while aging and dehydration are associated with decreased glucose tolerance, weight loss can improve glycemia. For older adults following hypocaloric diets, additional water consumption may lead to greater weight loss. Further more, research suggests an association between insulin resistance and the body water retention hormone, arginine vasopressin (AVP). Analysis of the association between plasma copeptin (an AVP derivative) and fasting glucose, insulin, and homeostasis model assessment of insulin resistance (HOMAIR) may provide further insight into the relationship between dehydration and diabetes risk. Our objective was to investigate the possibility that increased water consumption among older adults $\left(\mathrm{n}=29, \mathrm{BMI}=31 \pm 1 \mathrm{~kg} / \mathrm{m}^{2}\right.$, age $=62 \pm 1$ years) could improve glucose homeostasis beyond that observed with weight loss, as well as associations between plasma copeptin and diabetes risk. This retrospective analysis utilized data from a previous investigation, in which obese/overweight older adults were assigned to one of two groups: 1) Water: consume $500 \mathrm{ml}$ of water prior to three daily meals over a 12-week hypocaloric diet intervention, or 2) Non-water: hypocaloric diet alone. In the present analysis, fasting plasma glucose and insulin, HOMA-IR, and plasma copeptin were evaluated, and compared to urinary specific gravity (USG), drinking water consumption, and body weight. Analyses performed using group assignment, volume of drinking water consumed or among a subgroup pair-matched for weight loss and sex did not reveal significant differences between groups. However in the full sample, plasma insulin concentration was associated with USG $(r=0.512$, $P$ $<0.01)$ and copeptin $(r=0.389, P<0.05)$, and HOMA-IR was associated with USG $(r=0.530, P$ $<0.01$ ) at week 12 . Improvements in fasting insulin for water group participants $(-8.5+/-4$ $\mathrm{pmol} / \mathrm{L})$ were also detected. Associations between hydration and insulin resistance support the need for future investigations addressing hydration status and diabetes risk.

Keywords: Older Adults; Diabetes; Glucose; Water Consumption; Hydration

\section{INTRODUCTION}

The prevalence of diabetes for Americans aged 65+ years is about $30 \%$, and $50 \%$ of those in this age group are considered prediabetic $[1,2]$. Diabetes and prediabetes are major risk factors for cardiovascular and kidney diseases, thus intervention strategies aimed at reducing diabetes risk factors in this population are critically needed. Weight loss and increasing water consumption are lifestyle strategies which improve fasting glucose concentration and insulin resistance [3-7].

For the elderly population, adequate fluid intake and hydration status become especially important as decreased renal function leads to impairments in renalconcentrating and sodium-conserving ability, conditions associated with volume depletion and hypernatremia [8]. 
These conditions normally prompt thirst and subsequent fluid intake to restore hydration status, but thirst is often blunted in elderly subjects [8]. Hypodipsia (i.e., abnormally diminished thirst) in the elderly population is well established, but the underlying physiological mechanisms remain unclear. Under normal conditions, the feedback mechanisms of osmotic and baroreceptor pathways maintain proper water balance: elevated osmolarity and hypovolemia stimulate thirst and sodium appetite (to increase water intake), and secretion of arginine vasopressin (AVP) for an antidiuretic effect in the kidneys [9]. Dysfunctions within these systems are among the proposed mechanisms for decreased thirst in the elderly.

Older adults do not appear to be meeting beverage intake recommendations, and up to $40 \%$ of older adults may be chronically dehydrated [10]. Trends for total beverage intake by age indicate a sharp decrease for adults over 60 years old (average $2.1 \mathrm{~L} /$ day) [11]. Rates of plain water consumption also decrease with age (per- sons over 60 average only $0.73 \mathrm{~L} /$ day and $0.83 \mathrm{~L} /$ day for males and females, respectively) [12]. Inadequate water consumption can lead to disruptions in hydration status, placing older individuals at risk for numerous health complications (i.e. impaired cognitive function and motor control, increased resting heart rate, increased risk of infection, kidney and gall stone formation, higher incidence of colon and bladder cancer, heart arrhythmias, blood clots, and mitral valve prolapse) [8]. An inverse association between water intake and the development of hyperglycemia in adults has been reported [3], suggesting benefits of water consumption on blood glucose regulation.

Recent research suggests that the AVP system plays a role in glucose homeostasis, insulin resistance, and diabetes mellitus [13]. Copeptin is the c-terminal moiety of the AVP prohormone, and is cosecreted with vasopressin [14]. AVP is unstable, platelet-bound, rapidly cleared from the bloodstream, and difficult to measure reliably with available sandwich immunoassays [15]. Research shows that plasma copeptin correlates well with AVP release, and is therefore an acceptable alternative to direct measurement of AVP [15]. Higher copeptin concentrations have been reported among individuals with diabetes compared to non-diabetics, as well as positive associations between copeptin, fasting blood glucose, and plasma insulin in those without diabetes. Higher copeptin concentrations have also been reported at baseline for normoglycemic individuals who subsequently developed new-onset diabetes [13]. Positive correlations of plasma copeptin with components of metabolic syndrome (BMI, waist circumference, fasting serum glucose and insulin levels, HOMA-IR, presence of diabetes, and serum triglycerides) have also been reported [16]. Copeptin may therefore be associated with the presence of meta- bolic syndrome and may be a predictor for diabetes de- velopment. Furthermore, the elderly often have elevated plasma copeptin concentrations, indicating a decline in renal function and/or reduced sensitivity to AVP; researchers suggest this population may profit from interventions geared toward increasing water intake [17].

Weight loss is widely accepted as an effective approach for delaying or preventing type 2 diabetes [5-7]. Non-diabetic overweight and obese adults who replaced caloric beverages with water improved fasting glucose and hydration status, despite similar weight loss than a control group [4], indicating that drinking more water (regardless of weight loss) may influence glucose homeostasis.

A $500 \mathrm{ml}$ water preload consumed prior to each meal resulted in an approximate 13\% reduction in energy intake (EI) for overweight and obese older adults [18]. This reduced EI was sustained over a 12-week weight loss intervention as participants assigned to the water condition showed a $44 \%$ greater rate of weight loss (approximately $2 \mathrm{~kg}$ greater weight loss) than did those in the non-water group [19]. Augmenting weight loss with increased water consumption may have the potential to reduce the incidence of and progression to type 2 diabetes for overweight adults with diminished glucose control. To our knowledge, there are no studies which have directly addressed the possibility that increasing water intake in combination with weight loss in the older adult population will lead to improvements in fasting glucose and reductions in diabetes risk factors, or the exact mechanisms behind this additive effect. Available re- search has suggested beneficial effects on fasting glucose concentrations, but has not directly evaluated other dia- betes-related risk factors such as insulin resistance. Our objective was to determine the effects of weight loss in combination with increased water consumption on glucose homeostasis in older adults.

\section{MATERIALS AND METHODS}

This retrospective analysis utilized data collected previously [19], which did not include an analysis of diabetes risk factors. The randomized controlled trial was designed to determine if premeal water consumption (500 $\mathrm{ml}$ before each main meal, three times per day) would facilitate weight loss in overweight and obese older adults over 12 weeks. Participants were overweight or obese (BMI $\left.=25-40 \mathrm{~kg} / \mathrm{m}^{2}\right)$, between the ages of $55-$ 75 years, weight stable $(+2 \mathrm{~kg},>1$ year), and non-smokers. Assessments included height, measured in meters without shoes using a wall mounted stadiometer; weight, measured in light clothing and no shoes to the nearest 0.1 $\mathrm{kg}$ on a digital scale (Scale-Tronix model 5002, Wheaton, IL); dietary intake [19]; and urine, collected over 24 
hours for assessment of total volume, and urine specific gravity (USG) using a refractometer (Fisher UriSystem; Fisher Scientific, Hampton, NH). Although not reported in the original article, venous blood drawn in a fasted state was used to determine fasting plasma glucose (measured with a YSI glucose analyzer, model 2300, Yellow Springs Instruments) and insulin concentration (quantified using a commercially available ELISA, Linco Research, Inc.). Following completion of baseline assessments, participants were randomly assigned to one of two groups for 12 weeks: 1) hypocaloric diet $+500 \mathrm{ml}$ water prior to each daily meal (water group), or 2) hypocaloric diet alone (non-water group). Participants' body weight was measured weekly in the laboratory, and daily premeal water consumption logs were submitted by the water group at this time to monitor compliance. For the present investigation, plasma copeptin was assessed at baseline and at week 12 of the weight loss intervention, using an enzyme immunoassay (EIA) kit (Phoenix Pharmaceuticals Cat \#EK-065-32), and insulin resistance was estimated using homeostasis model assessment (HOMA) and an insulin resistance score (HOMA-IR) was computed with the following formula: fasting plasma glucose $(\mathrm{mmol} / \mathrm{L})$ times fasting serum insulin $(\mathrm{mU} / \mathrm{L})$ divided by 22.5 [20]. High HOMA-IR values indicate low insulin sensitivity (insulin resistance), and low HOMA-IR values indicate high sensitivity to insulin [20].

Statistical analyses were performed using SPSS statistical analysis software (v. 12.0). Independent sample ttests were used to assess group differences at baseline and differences in outcomes over time. One-sample ttests were used to assess pre-to-post change values within groups. Pearson correlation coefficients (r) meas- ured the strength of association between variables at baseline and 12 weeks, and associations between chan- ges in variables over the course of the intervention. Group differences in weight loss were noted in the origi- nal trial; therefore a pair-matched analysis was per- formed in a subset with participants paired according to both sex and total $\mathrm{kg}$ weight lost to eliminate these vari- ables as potential confounding factors. Six individuals from the water group (4 females, 2 males) were matched to individuals in the non-water group who had lost a similar amount of weight $(<0.6 \mathrm{~kg}$ difference between paired subjects). Paired samples t-tests were performed to determine paired differences for fasting glucose, insu- lin, HOMA-IR, and copeptin concentrations.

\section{RESULTS}

Of those who completed the original study, 29 had complete plasma glucose, insulin, and HOMA-IR results available, and only these participants were included in the present investigation. This subset consisted of overweight adults $\left(\mathrm{BMI}=31+1 \mathrm{~kg} / \mathrm{m}^{2}\right.$, age $=62+1$ years $)$ with 13 individuals assigned to the water group and 16 assigned to the non-water group.

There were no baseline group differences with respect to age, BMI, body weight, drinking water consumed, USG, fasting glucose or insulin concentration, HOMAIR, or plasma copeptin concentration. Water group participants reduced BMI, body weight, USG, and fasting insulin and increased water consumption at the end of the intervention period (all $\mathrm{P}<0.05$ ). Non-water group participants reduced BMI, body weight, and plasma copeptin concentration at the conclusion of the intervention (all $\mathrm{P}<0.05$ ). No group differences were noted in pre-to-post changes in BMI, body weight, USG, fasting glucose or insulin concentration, HOMA-IR score, or plasma copeptin concentration (Table 1). However, several notable correlations were detected. At baseline, body weight correlated with drinking water consumed $(\mathrm{r}=$ $-0.512, \mathrm{P}<0.01)$; plasma copeptin correlated with USG $(\mathrm{r}=0.424)$ and total grams of beverages consumed $(\mathrm{r}=$ $-0.403)(\mathrm{P}<0.05)$; fasting insulin correlated with grams of drinking water consumed $(\mathrm{r}=-0.378, \mathrm{P}<0.05)$; and HOMA-IR score correlated with USG $(\mathrm{r}=0.394, \mathrm{P}<$ $0.05)$ and drinking water consumed $(\mathrm{r}=-0.373, \mathrm{P}<$ 0.05). At week-12, plasma insulin correlated with USG ( $\mathrm{r}$ $=0.512, \mathrm{P}<0.01)$ and copeptin $(\mathrm{r}=0.389, \mathrm{P}<0.05)$; and HOMA-IR score correlated with USG $(\mathrm{r}=0.530, \mathrm{P}$ $<0.01)$.

In the full sample, participants in the lowest water consuming category ( $<500 \mathrm{~g} /$ day) were compared to the highest category $(>1000 \mathrm{~g} /$ day $)$, but no significant differences in outcomes were detected despite large differences in the means for fasting insulin $(-0.3+5$ vs. -9.2 $+3 \mathrm{pmol} / \mathrm{L}, \mathrm{P}=0.142$ ) (Table 2). In the sex and weight loss pair-matched sample, no differences were detected between water and non-water groups for fasting glucose $(83.9+7$ vs. $85.7+4 \mathrm{mg} / \mathrm{dl}, \mathrm{P}=0.801)$, fasting insulin $(29.2+6$ vs. $28.2+10 \mathrm{pmol} / \mathrm{L}, \mathrm{P}=0.923)$, HOMA-IR score $(0.84+0.1$ vs. $0.88+0.3, \mathrm{P}=0.888)$, or plasma copeptin $(3.8+1$ vs. $5.3+2 \mathrm{ng} / \mathrm{ml}, \mathrm{P}=0.598)$ at the conclusion of the intervention.

\section{DISCUSSION}

Despite existing data which supports the benefit of increased water consumption and weight loss on diabetes risk in older adults, our analyses did not demonstrate differences between intervention groups. However, associations between hydration status, fasting insulin concentrations and insulin resistance suggest that additional investigation is needed to determine the influence of hydration status combined with weight loss on glucose ho-meostasis and diabetes risk. Our findings are consis- 
Table 1. Participant characteristics and variables associated with weight loss, water consumption, and diabetes risk $(n=29)$.

\begin{tabular}{|c|c|c|c|c|c|c|}
\hline & \multicolumn{3}{|c|}{ Water Group Mean $\pm \operatorname{SEM}(\mathrm{n}=13)$} & \multicolumn{3}{|c|}{ Non-Water Group Mean \pm SEM $(n=16)$} \\
\hline & Baseline & Week 12 & Change & Baseline & Week 12 & Change \\
\hline \multicolumn{7}{|l|}{ Variables } \\
\hline Age, years & $62 \pm 1$ & ---- & ---- & $62 \pm 1$ & ---- & ---- \\
\hline BMI, $\mathrm{kg} / \mathrm{m}^{2 *}$ & $31.3 \pm 1$ & $28.9 \pm 1$ & $-2.4 \pm 0.4$ & $29.9 \pm 1$ & $28.4 \pm 1$ & $-1.4 \pm 0.3$ \\
\hline Weight, $\mathrm{kg}^{*}$ & $89.7 \pm 4$ & $82.8 \pm 4$ & $-7.0 \pm 1$ & $86.1 \pm 4$ & $81.9 \pm 4$ & $-4.2 \pm 1$ \\
\hline Drinking Water Consumed, $\mathrm{g}^{* *}$ & $359 \pm 87$ & $1307 \pm 154$ & $949 \pm 171^{*}$ & $501 \pm 142$ & $361 \pm 110$ & $-140 \pm 145$ \\
\hline Urinary Specific Gravity, UG & $1 \pm 0.001$ & $1 \pm 0.002$ & $-0.003 \pm 0.001^{*}$ & $1 \pm 0.001$ & $1 \pm 002$ & $-0.0002 \pm 0.001$ \\
\hline Fasting Glucose, mg/dl & $87.8 \pm 4$ & $86.2 \pm 4$ & $-1.5 \pm 3$ & $88.7 \pm 4$ & $87.2 \pm 4$ & $-1.5 \pm 2$ \\
\hline Fasting Insulin, pmol/L & $39.0 \pm 4$ & $30.5 \pm 4$ & $-8.5 \pm 4^{*}$ & $35.2 \pm 5$ & $34.6 \pm 6$ & $-0.6 \pm 3$ \\
\hline HOMA-IR Score & $1.2 \pm 0.1$ & $0.9 \pm 0.1$ & $-0.3 \pm 0.1$ & $1.1 \pm 0.1$ & $1.1 \pm 0.2$ & $-0.01 \pm 0.1$ \\
\hline Plasma Copeptin, ng/ml & $3.7 \pm 0.5$ & $3.9 \pm 1$ & $0.2 \pm 0.8$ & $4.5 \pm 1$ & $3.5 \pm 0.9$ & $-1 \pm 0.4^{*}$ \\
\hline
\end{tabular}

*Significant differences detected from baseline to week 12 within groups $(\mathrm{P}<0.05) ;{ }^{* *}$ Significant differences detected from baseline to week 12 between groups $(\mathrm{P}<0.05)$.

Table 2. Group differences according to two drinking water categories at week 12 of the intervention $(n=25)$.

\begin{tabular}{ccc}
\hline & \multicolumn{1}{c}{ Drinking Water Categories at Week 12 } \\
\hline Variables & $<\mathbf{5 0 0}$ g/day Mean \pm SEM $(\mathrm{n}=13)$ & $>\mathbf{1 0 0 0}$ g/day Mean \pm SEM $(\mathrm{n}=12)$ \\
Weight Lost, $\mathrm{kg}$ & $-4.7 \pm 1$ & $-7.3 \pm 1$ \\
Change BMI, $\mathrm{kg} / \mathrm{m}^{2}$ & $-1.6 \pm 0.4$ & $-2.6 \pm 0.5$ \\
Change Body Fat, $\mathrm{kg}$ & $-3.5 \pm 0.8$ & $-4.6 \pm 0.9$ \\
Change Fasting Glucose, $\mathrm{mg} / \mathrm{dl}$ & $-1.7 \pm 3$ & $-2.9 \pm 2$ \\
Change Fasting Insulin, $\mathrm{pmol} / \mathrm{L}$ & $-0.3 \pm 5$ & $-9.2 \pm 3$ \\
Change HOMA-IR Score & $-0.01 \pm 0.2$ & $-0.3 \pm 0.1$ \\
Change Plasma Copeptin, $\mathrm{ng} / \mathrm{ml}$ & $-0.5 \pm 0.8$ & $-0.2 \pm 0.5$ \\
\hline
\end{tabular}

tent with previous research which has reported positive correlations of plasma copeptin concentration with components of metabolic syndrome, which included fasting insulin levels and HOMA-IR.

According to available literature, men tend to have higher plasma copeptin levels than women [15,21,22]. In this investigation, no gender differences were detected among the full sample for plasma copeptin at baseline, week 12 , or pre-to-post changes, which led to the pairmatch based upon both weight loss and sex. Limitations exist with regard to the copeptin analysis, as copeptin is influenced by recent water consumption and fasting and therefore may not reflect chronic hydration status. However, in our analysis, copeptin concentration was inversely associated with self-reported fluid consumption according to four-day food and beverage intake records, and positively associated with USG. The clinical significance of plasma copeptin as a diagnostic tool is still un- der investigation, and further research into its relevance is warranted.

Although the small sample size likely limited the statistical power to detect differences in diabetes risk factors between groups, this preliminary study may be used to determine sample sizes needed for future investigations. Successful approaches to loss and maintenance of body weight are essential for lowering risk factors for diabetes, and additional studies are needed to determine if health messages and clinical practice should be altered to include recommendations on increasing water consumption in conjunction with weight loss for the older adult population. Adding water intake recommendations to common healthcare practice may therefore result in improved health outcomes with relation to hydration, as well as diabetes risk for older adults. Our intervention approach may represent a feasible and potentially effective approach for reducing the prevalence and progression to 
diabetes for at risk older adults.

\section{REFERENCES}

[1] CDC (2011) National Diabetes Fact Sheet: National estimates and general information on diabetes and prediabetes in the United States. US Department of Health and Human Services, Centers for Disease Control and Prevention.

[2] Eikenberg, J.D. and Davy, B.M. (2013) Prediabetes: A prevalent and treatable, but often unrecognized, clinical condition. Journal of the Academy of Nutrition and Dietetics, 113, 213-218.

[3] Roussel, R.F.L., Bouby, N., Balkau, B., Lantieri, O., Alhenc-Gelas, F., Marre, M. and Bankir, L. (2011) Low water intake and risk for new-onset hyperglycemia American Diabetes Association. Diabetes Care, 34, 2551-2554.

[4] Tate, D.F., Turner-McGrievy, G., Lyons, E., Stevens, J., Erickson, K., Polzien, K., et al. (2012) Replacing caloric beverages with water or diet beverages for weight loss in adults: main results of the Choose Healthy Options Consciously Everyday (CHOICE) randomized clinical trial. The American Journal of Clinical Nutrition, 95, 555-563.

[5] Colman, E., Katzel, L.I., Rogus, E., Coon, P., Muller, D. and Goldberg, A.P. (1995) Weight loss reduces abdominal fat and improves insulin action in middle-aged and older men with impaired glucose tolerance. Metabolism, 44, 1502-1508.

[6] Tuomilehto, J., Lindstrom, J., Eriksson, J.G., Valle, T.T., Hamalainen, H., Ilanne-Parikka, P., et al. (2001) Prevention of type 2 diabetes mellitus by changes in lifestyle among subjects with impaired glucose tolerance. The New England Journal of Medicine, 344, 1343-1350.

[7] Knowler, W.C., Barrett-Connor, E., Fowler, S.E., Hamman, R.F., Lachin, J.M., Walker, E.A., et al. (2002) Reduction in the incidence of type 2 diabetes with lifestyle intervention or metformin. The New England Journal of Medicine, 346, 393-403.

[8] (2005) Dietary reference intakes for water, potassium, sodium, cholride, and sulfate: Chapter 4 water. The National Academies Press, Washington, D.C.

[9] Stipanuk, M. and Caudill, M. (2013) Biochemical, physiological, and molecular aspects of human nutrition. 3rd Edition, Elsevier,

[10] Stookey, J.D. (2005) High prevalence of plasma hypertonicity among community-dwelling older adults: Results from NHANES III. Journal of the American Dietetic Association, 105, 1231-1239.

[11] Popkin, B.M. (2010) Patterns of beverage use across the lifecycle. Physiology \& Behavior, 100, 4-9.
[12] US Department of Agriculture (2011) What we eat in america, national health and nutrition examination survey 2005-2008, drinking water intake in the US. Dietary Data Brief No. 7.

[13] Enhorning, S., Wang, T.J., Nilsson, P.M., Almgren, P., Hedblad, B., Berglund, G., et al. (2010) Plasma copeptin and the risk of diabetes mellitus. Circulation, 121, 2102 2108.

[14] Balanescu, S., Kopp, P., Gaskill, M.B., Morgenthaler, N.G., Schindler, C. and Rutishauser, J. (2011) Correlation of plasma copeptin and vasopressin concentrations in hypo-, iso-, and hyperosmolar States. The Journal of Clinical Endocrinology \& Metabolism, 96, 1046-1052.

[15] Morgenthaler, N.G., Struck, J., Alonso, C. and Bergmann, A. (2006) Assay for the measurement of copeptin, a stable peptide derived from the precursor of vasopressin. Clinical Chemistry, 52, 112-119.

[16] Saleem, U., Khaleghi, M., Morgenthaler, N.G., Bergmann, A., Struck, J., Mosley, T.H., Jr., et al. (2009) Plasma carboxy-terminal provasopressin (copeptin): A novel marker of insulin resistance and metabolic syndrome. The Journal of Clinical Endocrinology \& Metabolism, 94, 25582564.

[17] Meijer, E., Bakker, S.J., de Jong, P.E., Homan van der Heide, J.J., van Son, W.J., Struck, J., et al. (2009) Copeptin, a surrogate marker of vasopressin, is associated with accelerated renal function decline in renal transplant recipients. Transplantation, 88, 561-567.

[18] Davy, B.M., Dennis, E.A., Dengo, A.L., Wilson, K.L. and Davy, K.P. (2008) Water consumption reduces energy intake at a breakfast meal in obese older adults. Journal of the American Dietetic Association, 108, 1236-1239.

[19] Dennis, E.A., Dengo, A.L., Comber, D.L., Flack, K.D., Savla, J., Davy, K.P., et al. (2010) Water consumption increases weight loss during a hypocaloric diet intervention in middle-aged and older adults. Obesity, 18, 300-307.

[20] Bonora, E., Formentini, G., Calcaterra, F., Lombardi, S., Marini, F., Zenari, L., et al. (2002) HOMA-estimated insulin resistance is an independent predictor of cardiovascular disease in type 2 diabetic subjects: Prospective data from the Verona Diabetes Complications Study. Diabetes Care, 25, 1135-1141.

[21] Bhandari, S.S., Loke, I., Davies, J.E., Squire, I.B., Struck, J. and $\mathrm{Ng}$, L.L. (2009) Gender and renal function influence plasma levels of copeptin in healthy individuals. Clinical Science (Lond), 116, 257-263.

[22] Morgenthaler, N.G., Struck, J., Jochberger, S. and Dunser, M.W. (2008) Copeptin: Clinical use of a new biomarker. Trends in Endocrinology \& Metabolism, 19, 43-49. 